\title{
Accelerated Carrier Relaxation through Reduced Coulomb Screening in Two- Dimensional Halide Perovskite Nanoplatelets
}

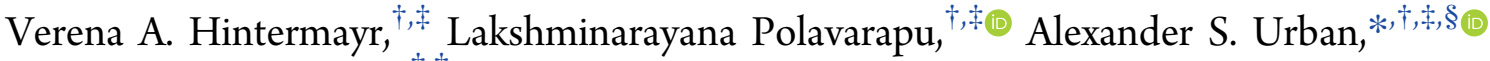
and Jochen Feldmann*, ${ }^{*}$,

${ }^{\dagger}$ Chair for Photonics and Optoelectronics, Department of Physics, Ludwig-Maximilians-Universität München, Amalienstr. 54, 80799 Munich, Germany

${ }^{\ddagger}$ Nanosystems Initiative Munich (NIM) and Center for NanoScience (CeNS), Schellingstr. 4, 80799 Munich, Germany

\section{Supporting Information}

ABSTRACT: For high-speed optoelectronic applications relying on fast relaxation or energy-transfer mechanisms, understanding of carrier relaxation and recombination dynamics is critical. Here, we compare the differences in photoexcited carrier dynamics in two-dimensional (2D) and quasi-threedimensional (quasi-3D) colloidal methylammonium lead iodide perovskite nanoplatelets via differential transmission spectroscopy. We find that the cooling of excited electron-hole pairs by phonon emission progresses much faster and is intensityindependent in the $2 \mathrm{D}$ case. This is due to the low dielectric surrounding of the thin perovskite layers, for which the Fröhlich interaction is screened less efficiently leading to higher and less density-dependent carrier-phonon scattering rates. In addition, rapid dissipation of heat into the surrounding occurs due to the high surface-to-volume ratio. Furthermore, we observe a subpicosecond dissociation of resonantly excited $1 \mathrm{~s}$ excitons in the quasi-3D case, an effect which is suppressed in the $2 \mathrm{D}$ nanoplatelets due to their large exciton binding energies. The results highlight the importance of the surrounding environment of the inorganic nanoplatelets on their relaxation dynamics. Moreover, this 2D material with relaxation times in the subpicosecond regime shows great potential for realizing devices such as photodetectors or all-optical switches operating at $\mathrm{THz}$ frequencies.

KEYWORDS: perovskite, nanoplatelets, Coulomb screening, carrier relaxation, transient absorption spectroscopy, Fröhlich interaction
$\mathrm{L}$ ead halide perovskite thin films have established themselves as an excellent material system for photovoltaic applications due to favorable absorption and charge transport properties. $^{1-4}$ With bandgaps tunable throughout the visible range via halide ion replacement ${ }^{5,6}$ and quantum yields approaching unity, perovskite nanocrystals (NCs) exhibit strong potential for a variety of other optoelectronic applications. ${ }^{7-10}$ While in both of these fields either bulk or only weakly confined perovskite materials have been employed, recently strongly confined perovskite NCs, such as two-dimensional (2D) nanoplatelets (NPls), with a monolayer-precise control of the thickness and resulting quantum size effects have emerged. ${ }^{11-15}$ With extremely high exciton binding energies and transition energies tunable through quantum confinement, they demonstrate intriguing possibilities for light-emitting applications such as LEDs or lasers. $^{16-18}$ Moreover, with excitons dominant at roomtemperature perovskite NPls are also promising for excitonic device concepts. Importantly, high-frequency limitations depend on the time scales for relaxation and recombination scenarios of photoexcited or electrically injected charge carriers.

In this paper, we investigate charge carrier relaxation in $2 \mathrm{D}$ and quasi-three-dimensional (quasi-3D) methylammonium lead iodide (MAPI) NPls by means of femtosecond differential transmission spectroscopy (DTS) at room temperature. Linear absorption spectroscopy initially reveals the effect of quantum confinement on the continuum absorption onset position and on the exciton binding energy, which is found to be more than 10 times larger for the 2D NPls as compared to the quasi-3D NPls. Consistently, results of transient DTS measurements show a bimolecular recombination due to free electron-hole pairs for the quasi-3D NPls and a monomolecular decay behavior characteristic for excitonic decay for the 2D NPls.

Received: July 3, 2018

Accepted: October 8, 2018

Published: October 8, 2018 

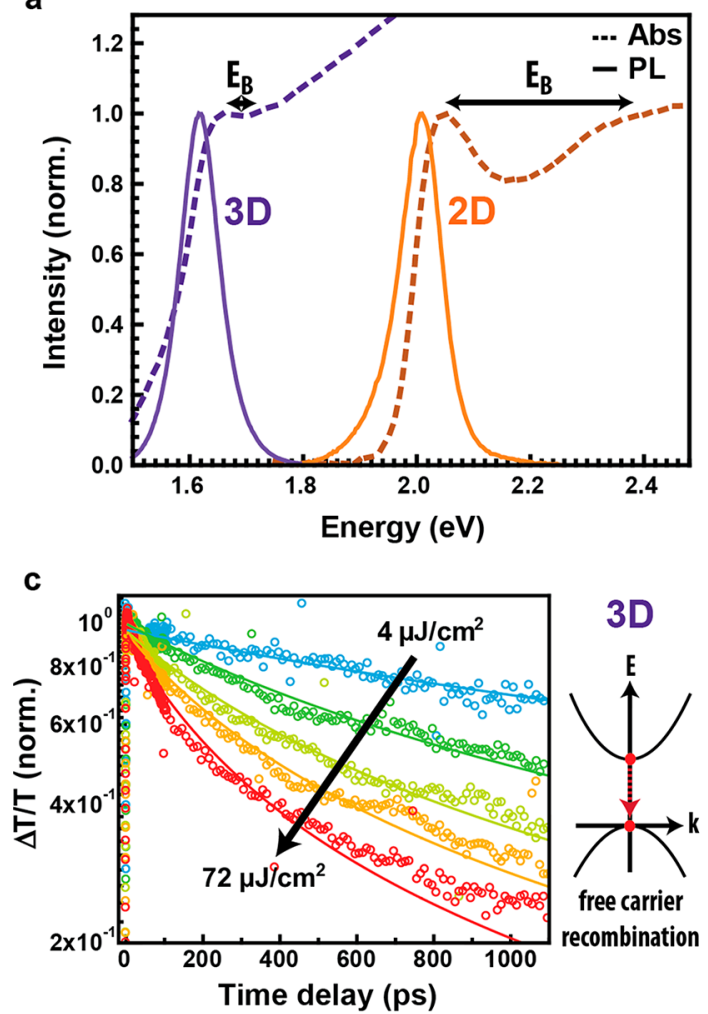

b

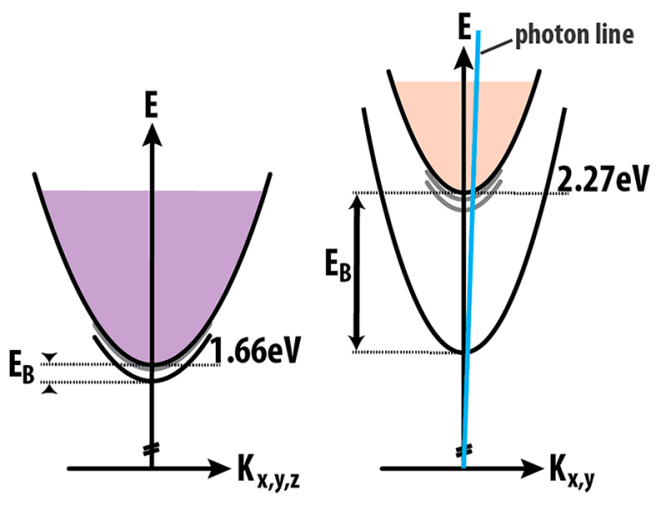

d

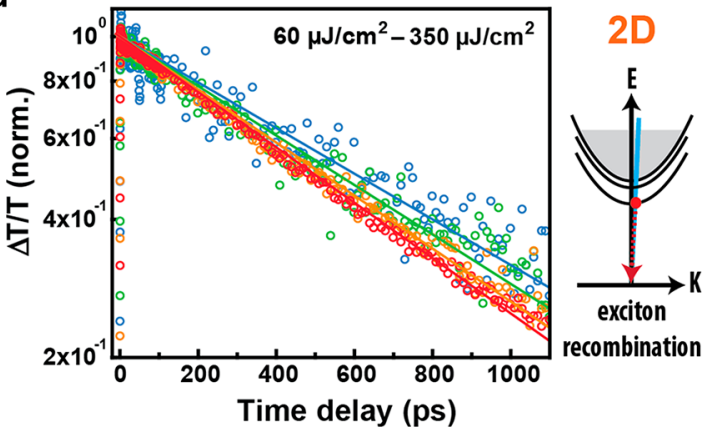

Figure 1. Energetics and charge carrier recombination of 2D and quasi-3D MAPI NPIs. (a) Steady-state absorption and PL spectra of quasi3D (purple) and 2D (orange) NPls. The corresponding values of the exciton binding energies $E_{\mathrm{b}}$ are shown as arrows in the absorption spectra. (b) Dispersion relations $E(K)$ for bound and unbound electron-hole pairs. Within this two-particle picture $K$ represents the wave vector for the center-of-mass motion of electron-hole pairs. (c, d) Transients of the $\Delta T / T$ signals at the 1s exciton for the quasi-3D (c) and 2D case (d) as a function of laser excitation density (here shown for a monolayer nanoplatelet). The solid lines are the results of calculated transients assuming bimolecular decay kinetics $\left(\Delta T \sim n_{\mathrm{e}} \times n_{\mathrm{h}}\right)$ and monomolecular decay kinetics $(\Delta T \sim n)$ for the 3D and 2D case, respectively. On the right side, the corresponding recombination process is depicted for the quasi-3D in the one-particle (c) and for the $2 \mathrm{D}$ case in the two-particle (d) picture, respectively.

Regarding the initial carrier relaxation, we find an intensityindependent subpicosecond cooling time in the $2 \mathrm{D} \mathrm{NPls}$ which is seven times faster than the strongly intensitydependent cooling time in the quasi-3D NPls. This can be explained by the large surface-to-volume ratio of the $2 \mathrm{D} \mathrm{NPls}$ hindering the creation of hot phonons and a less efficient screening of the photoexcited carriers, leading to high excitonoptical phonon scattering rates, governed by the Fröhlich interaction. $^{19,20}$ Finally, by comparing resonant and nonresonant pump pulse excitation of the $1 \mathrm{~s}$ exciton transitions, we directly monitor the exciton dissociation for the quasi-3D case, whereas resonantly excited excitons in the 2D NPls stay on the $1 \mathrm{~s}$ exciton parabola. Our findings highlight the vast differences occurring in perovskite materials based on their dimensionality. Knowledge of these processes could prove critical to designing optoelectronic devices relying on fast relaxation as well as charge- or energy-transfer mechanisms.

\section{RESULTS}

Confinement Effects and Exciton Binding Energies. MAPI NPls were synthesized by ligand-assisted exfoliation, as previously developed by our group and as detailed in the Methods section. ${ }^{12}$ Their thickness is quantized according to an integer number of $2 \mathrm{D}$ layers of corner-sharing $\left[\mathrm{PbI}_{6}\right]^{-4}$ octahedra down to a single monolayer $(0.66 \mathrm{~nm})$. Resulting $\mathrm{NPl}$ dispersions can be used to study the effect of quantum confinement in one dimension, as the thick bulk-like NPls are progressively shrunk down to the limit of $2 \mathrm{D}$ NPls. To show the difference between $2 \mathrm{D}$ and $3 \mathrm{D} \mathrm{NPls}$, we selected a dispersion of trilayer MAPI NPls (thickness $d \approx 2 \mathrm{~nm}$ ) and thicker NPls $(d \geq 15 \mathrm{~nm})$, which are referred to as quasi-3D NPls, due to their bulk-like optical properties (see also Supporting Information, Figures S1 and S2). The thickness of the MAPI trilayer is in the range of the $3 \mathrm{D}$ excitonic Bohr radius of $2.5 \mathrm{~nm}$ and thus can be considered as quantum confined in one dimension, whereas the bulk-like NPls are unconfined or only weakly confined, hence justifying the distinction as $2 \mathrm{D}$ and quasi-3D NPls, respectively. ${ }^{21}$

All measurements presented here were conducted at room temperature on dispersions of colloidal NPls in toluene. In Figure 1a, linear absorption and photoluminescence (PL) spectra are shown for the $2 \mathrm{D}$ and the quasi-3D NPls. The absorption spectrum in the $2 \mathrm{D}$ case reveals a $1 \mathrm{~s}$ exciton resonance at $2.0 \mathrm{eV}$ easily distinguishable from the onset of the electron-hole continuum transitions at $2.27 \mathrm{eV}$. For the quasi$3 \mathrm{D}$ case, the excitonic transitions spectrally overlap with the continuum absorption onset due to homogeneous and inhomogeneous broadening effects. ${ }^{22}$ Applying the Elliott model to the linear absorption spectra of the NPls ( $c f$. Figure S3) we obtain band gaps of $E_{\mathrm{g}}^{3 \mathrm{D}}=1.66 \mathrm{eV}$ and $E_{\mathrm{g}}^{2 \mathrm{D}}=2.27 \mathrm{eV}$ as well as exciton binding energies of $E_{\mathrm{b}}^{3 \mathrm{D}}=19 \mathrm{meV}$ and $E_{\mathrm{b}}^{2 \mathrm{D}}=$ $230 \mathrm{meV}$. These values correspond well to the range of previously published values and confirm the bulk-like nature of the thicker platelets. ${ }^{23,24}$ For the $3 \mathrm{D}$ NPls, the exact 
a

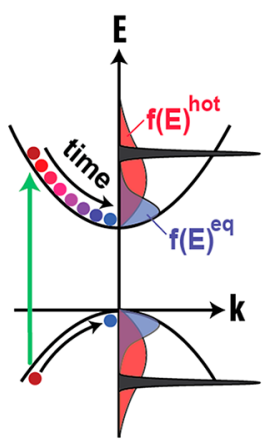

d

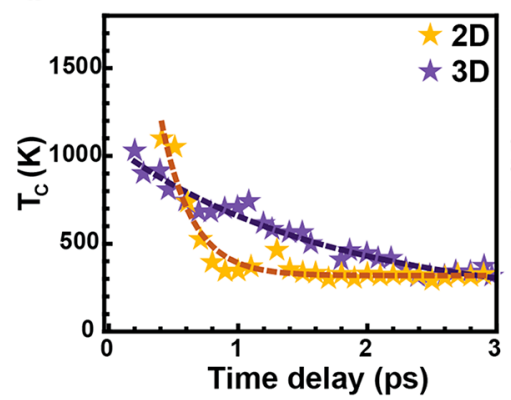

b

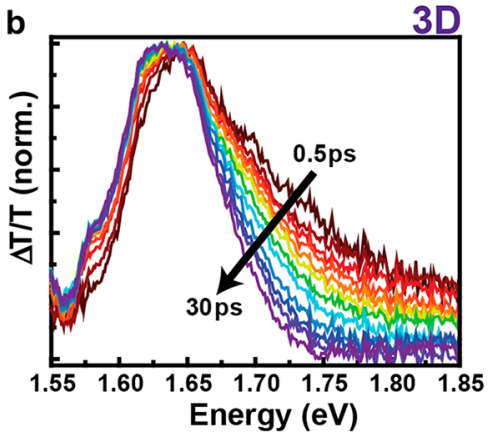

e

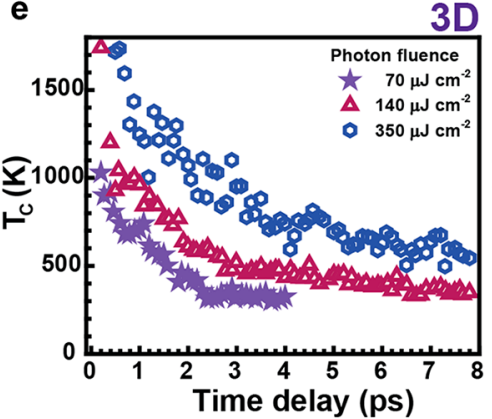

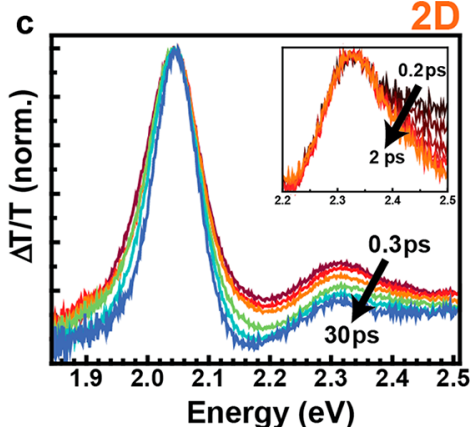

$f$

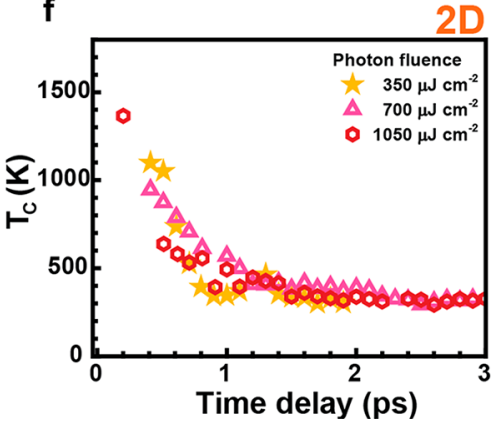

Figure 2. Photoexcited charge carrier cooling. (a) Scheme of the thermalization and relaxation of photoexcited electrons and holes in the one-particle picture. The initially independent $\delta$-like distribution functions are depicted for electrons in the conduction band and holes in the valence band. Directly after thermalization the carriers assume a (hot) thermal distribution $f(E)^{\text {hot }}($ red $) . f(E)^{\text {eq }}($ blue $)$ describes the distribution when the charge carriers have cooled down sufficiently to reach equilibrium with the crystal lattice. (b, $c$ ) Normalized $\Delta T$ spectra with increasing time delays from 0.3 to 30 ps for the quasi-3D (b) and $2 \mathrm{D}$ (c) case. In the inset of (c) the $\Delta T$ spectra are redrawn normalized to the values at the continuum absorption onset. (d) Transients of the carrier temperature $T_{\mathrm{c}}$ (cooling curves) as calculated from eq 3 for the quasi-3D and the 2D sample. The dashed lines represent calculated exponential decays with time constants of 1.7 ps and 240 fs, nicely resembling the experimentally obtained cooling curves. (e, f) Photon fluence-dependent measurements of the cooling curves for quasi-3D (e) and 2D (f) NPls.

determination of the binding energy is difficult due the broadening of the absorption spectrum, and so the obtained value constitutes an upper bound. ${ }^{25}$ Nevertheless, the exciton binding energy of the 2D NPLs is more than 10 times as large as in the $3 \mathrm{D}$ case. This is caused by a change in dimensionality (in the ideal case: $E_{\mathrm{b}}^{2 \mathrm{D}}=4 \cdot E_{\mathrm{b}}^{3 \mathrm{D}}$ ) and by a reduced screening of the Coulomb interaction between electron and hole due to the low dielectric constant of the organic ligands and solvent surrounding the inorganic NPls. ${ }^{8}$ Similar values for the exciton binding energy were already observed in the 1990s for layered PbI-based perovskite structures. ${ }^{26-28}$ The PL spectra of the $\mathrm{NPls}$ display single and narrow resonances at $E_{\mathrm{PL}}^{3 \mathrm{D}}=1.62 \mathrm{eV}$ and $E_{\mathrm{PL}}^{2 \mathrm{D}}=2.03 \mathrm{eV}$, respectively, exhibiting Stokes shifts of $<20$ $\mathrm{meV}$. This confirms the high quality and uniformity of the NPls. $^{11}$

These findings are represented schematically in Figure $1 \mathrm{~b}$, where the dispersion relations $E(K)$ for bound and unbound electron-hole pairs are depicted for the two perovskite NC systems. The huge blueshift of approximately $600 \mathrm{meV}$ of the energetic states due to quantum confinement in $z$-direction, dispersion in only two directions $(x, y)$, and the large exciton binding energy are the prominent characteristics of the 2D NPls. While for the quasi-3D system, the exciton binding energy $(19 \mathrm{meV})$ is smaller than the thermal energy $k T$ at room temperature $(\sim 26 \mathrm{meV})$, the value for the $2 \mathrm{D}$ system is far above it. Importantly, for 2D NPls, it is not enough just to consider quantum confinement as done for quantum dots, since apart from the confinement in $z$-direction, the dispersion in $\mathrm{x}$ - and $y$-directions is still retained; a fact which is necessary to understand the time-resolved optical experiments presented in this paper.

Photoinduced Carrier Dynamics. Time-resolved optical studies on relaxation and recombination scenarios of photoexcited electrons and holes in halide perovskite NPls have been restricted to PL to date. As indicated by the photon line for the $2 \mathrm{D}$ case in Figure $1 \mathrm{~b}$, in such nonresonant PL experiments, electron-hole pairs are typically excited far above the continuum edge and then relax down to the $1 \mathrm{~s}$ exciton parabola. Radiative emission finally takes place from the crossing point of the $1 \mathrm{~s}$ exciton parabola $E_{1 s}\left(K_{x, y}\right)$ with the photon line close to $K=0$. Due to a finite homogeneous line width, the radiative emission stems from a respective "radiative window" $\Delta E$ corresponding to an area $\Delta K$ in $K_{x, y}$-space and to a coherence area in real space, as theoretically introduced and experimentally demonstrated for the case of GaAs quantum wells $^{29}$ and recently also shown for II-VI NPls. ${ }^{30}$

Consequently, time-resolved PL experiments can provide nearly no information on the initial relaxation dynamics, which occurs before the electron-hole pairs reach the radiative window on the $1 \mathrm{~s}$ exciton parabola. Instead, transient absorption experiments with femtosecond pump and probe laser pulses have proven to be sensitive to the initial ultrafast dynamics of photoexcited electron-hole pairs. ${ }^{31}$ The photoexcitation by the pump pulse initially creates a coherent and highly nonthermal electron-hole distribution at the $E(K)$ position, where the photon line meets the continuum absorption for the given photon excitation energy (Figure 2a). This nonthermal distribution then thermalizes typically on a subpicosecond time-scale through carrier-carrier and carrier- 
phonon scattering. ${ }^{31,32}$ Thermalization means that thereafter the energetic distribution of electrons and holes can be described with Fermi-Dirac distribution functions $f_{\mathrm{e}, \mathrm{h}}\left(E, T_{\mathrm{c}}\right)$. The distribution is generally referred to as "hot", as carrier temperatures $T_{\mathrm{c}}$ can initially be higher than $1000 \mathrm{~K}$. Through phonon emission, these carrier distributions cool down until the charge carriers reach thermal equilibrium with the crystal lattice. With sufficient excess energy of the charge carriers, the initial cooling occurs within a few picoseconds due to Coulomb-mediated scattering with optical phonons (Fröhlich interaction). The cooling process typically slows down for higher excitation densities, as phonon reabsorption increases with increasing phonon density ("hot phonon effect"), ${ }^{33,34}$ and the high density of photoexcited carriers leads to a screening of the Fröhlich interaction. ${ }^{19,20,31,35}$ Once the carrier distribution has cooled so far that optical phonons cannot be emitted anymore $\left(k T_{\mathrm{c}} \lesssim E_{\mathrm{LO}}\right)$, the final cooling can only progress through less efficient acoustic phonon scattering, typically caused by deformation potentials. ${ }^{20,36}$ All of these relaxation and cooling processes influence the onset and (if slow enough) even the decay behavior of the 1 s exciton PL.

Recombination. To investigate the relaxation and recombination, we have employed transient DTS on the $2 \mathrm{D}$ and 3D NPls, exciting far above the continuum onset at 400 $\mathrm{nm}$, focusing initially on the decay of the $\Delta T$ signal for long time delays, $\tau$ between the pump and probe pulses. As shown in Figure $1 \mathrm{c}, \mathrm{d}$, the observed $\Delta T(\tau)$ transients for $2 \mathrm{D}$ and quasi-3D NPls behave differently. For the quasi-3D NPls, the decay at the position of maximum bleaching signal follows a $1 / \tau$ behavior, indicating bimolecular decay kinetics, and with $\Delta T \sim n_{\mathrm{e}} \times n_{\mathrm{h}}$ likely stemming from free carrier recombination. The decay becomes progressively faster as the excitation density is increased. In contrast, the decay behavior of the $\Delta T(\tau)$ transients at the position of the largest bleaching signal is purely exponential for $2 \mathrm{D} \mathrm{NPls}$ indicating monomolecular decay kinetics, and with $\Delta T \sim n$, likely excitonic recombination (Figure 1d). Excitonic PL transients of halide perovskite mono-, bi-, and trilayers also show such a monomolecular decay behavior as recently discussed by our group. ${ }^{12}$ This is a direct consequence of the significantly enhanced exciton binding energy in the $2 \mathrm{D} \mathrm{NPls,} \mathrm{which} \mathrm{impedes} \mathrm{a} \mathrm{thermal}$ dissociation of the exciton at room temperature. In contrast, in the quasi-3D NPls, with an exciton binding energy below the thermal energy at room temperature, free electrons and holes are predominant. In both cases as the excitation density is increased, the initial decay of the measured $\Delta T$ transients becomes slightly faster than described by the calculated transients. Auger recombination and exciton-exciton annihilation set in for the $3 \mathrm{D}$ and $2 \mathrm{D} \mathrm{NPls}$, respectively, as already reported for bulk halide perovskite films. ${ }^{23}$

Relaxation and Cooling. In order to address the relaxation and cooling dynamics of photoexcited electronhole pairs, we investigate the DTS signals at time delays between 300 fs and 30 ps. Normalized $\Delta T$ spectra are shown in Figure $2 \mathrm{~b}, \mathrm{c}$ for the quasi-3D and $2 \mathrm{D}$ cases with photon fluences of $350 \mu \mathrm{J} / \mathrm{cm}^{2}$ and $700 \mu \mathrm{J} / \mathrm{cm}^{2}$ per pump pulse, respectively. For all measurements, we assured that the change in absorption upon pumping $(\Delta A / A)$ remained below $10 \%$ for the two systems in order to be able to compare them and ensure that the systems are pumped weakly enough so that the transmission is roughly proportional to the charge carrier density and to remain far away from gain conditions. Apart from the main bleaching signal at the two respective 1s exciton energies ( 1.62 and $2.04 \mathrm{eV}$ ), additional transmission changes occur at higher photon energies. For the quasi-3D NPls these extra $\Delta T$ contributions start right on the high-energy side of the 1s exciton and exhibit an approximately exponential decay to higher energies (Figure $2 \mathrm{~b}$ ). This is a clear indication that hot electron-hole pair distributions bleach the continuum absorption by phase space filling. ${ }^{37}$ For the $2 \mathrm{D} \mathrm{NPls,} \mathrm{the}$ corresponding additional $\Delta T$ signal around $2.3 \mathrm{eV}$ is well separated from the $1 \mathrm{~s}$ exciton signal, as can be seen clearly in the inset in Figure 2c, showing the $\Delta T$ spectra normalized to the values at the continuum absorption onset. These results confirm our interpretation of the absorption spectra within the Wannier-Mott exciton model for the quasi-3D as well as the 2D NPls. For both cases, the distribution functions obviously cool down with increasing time delays $\tau$, so we can use the $\Delta T$ spectra above the continuum absorption onset for a given time delay to calculate the respective carrier temperature $T_{\mathrm{c}}$. We assume that the Fermi-Dirac distribution function $f_{\mathrm{FD}}\left(E, T_{\mathrm{c}}\right)$ can be approximated by a Boltzmann distribution function leading to 38,39

$$
\frac{\Delta T(\tau)}{T} \propto\left(-\frac{E-E_{\text {cont }}}{k_{\mathrm{B}} T_{\mathrm{c}}(\tau)}\right)
$$

In this way, we obtain transient cooling curves $T_{c}(\tau)$ for the quasi-3D and the 2D NPls as depicted in Figure $2 d-f$. To ensure that the carriers have thermalized, we only analyze cooling curves for time delays larger than $200 \mathrm{fs}^{32}$ Excited at the lowest photon fluences possible, the two samples both show initial charge carrier temperatures well above $1000 \mathrm{~K}$ as shown in Figure $2 \mathrm{~d}$. The charge carriers rapidly cool down in both systems due to emission of optical phonons, which can be modeled by assuming an exponential decay (see dashed lines in Figure $2 \mathrm{~d}$ ). For the quasi-3D system, we obtain a time constant of $1.7 \mathrm{ps}$, while in the $2 \mathrm{D}$ system, carriers cool down much more rapidly, namely with a time constant of $240 \mathrm{fs}$ (see Table S1 for all cooling times). Importantly, these times are significantly faster than the observed Auger recombination (Figure 1c) and also than Auger lifetimes reported on recently. ${ }^{40}$ Consequently, Auger recombination should not affect the charge carrier cooling despite the high laser fluences and can be neglected. This rapid cooling is not followed by a subsequent cooling due to the emission of acoustic phonons, since the thermal energy of the lattice at room temperature $\left(k T_{\mathrm{L}}=26 \mathrm{meV}\right)$ exceeds the energy of optical phonons in MAPI $\left(h v_{\mathrm{LO}}=12-17 \mathrm{meV}\right){ }^{41,42}$ That the cooling progresses faster in the $2 \mathrm{D}$ than in the quasi-3D case seems counterintuitive initially. As the dimensionality is reduced from $3 \mathrm{D}$ to $2 \mathrm{D}$, the number of phonon modes and the density of states (DOS) for electrons are reduced. Consequently, one would expect a reduced carrier-phonon scattering rate and therefore longer cooling times for the $2 \mathrm{D}$ system. Ultimately, the carrier confinement leads to a so-called phonon-bottleneck and thus slow cooling when the system is completely confined for quantum dots (OD). ${ }^{43-47}$ However, for the perovskite NPI system, another process leads to enhanced carrier scattering. Instead of being embedded within another semiconductor, the MAPI NPls with a large dielectric permittivity are passivated by organic ligands and immersed in an organic solvent-here toluene-with a small dielectric permittivity, as shown schematically in Figure 3. Consequently, Coulomb interactions with excited charge carriers in the quasi-3D perovskite NPls are screened by the large values of the permittivity, while those in 

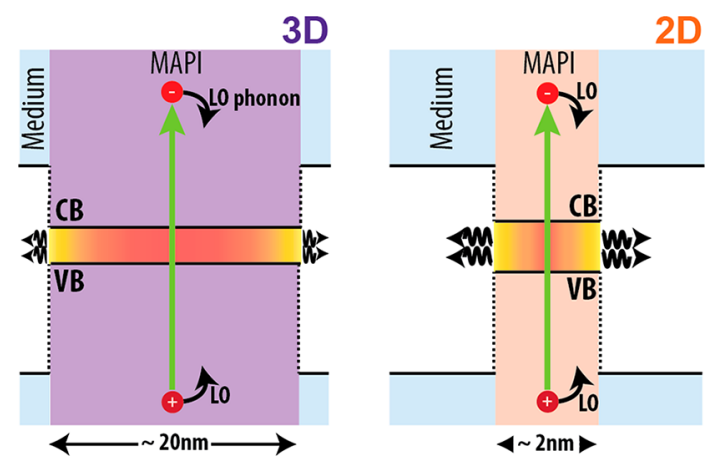

Figure 3. Scheme of carrier cooling processes in thick (quasi-3D) and thin (2D) NPls. After photoexcitation, the carriers cool down via emission of LO-phonons. As described in the main text, both screening effects and larger surface-to-volume ratios explain why in the $2 \mathrm{D}$ case, carrier cooling is faster and a dependence on carrier density is not observed.

the $2 \mathrm{D}$ perovskite NPls will be less screened due to the penetration of electric field lines into the surrounding low dielectric permittivity medium. This not only leads to the extremely large binding energies of excitons but also has a pronounced effect on the scattering of charge carriers among each other and with optical phonons, as governed by the Fröhlich interaction. ${ }^{33}$ The observed faster carrier cooling in 2D perovskite NPls is thus a direct consequence of the reduced screening of the Coulomb-mediated carrier-LO-phonon interaction in the low-dielectric surrounding. In turn, the more pronounced screening results in a slower cooling process of carriers in quasi-3D MAPI NPls.
Interestingly, as shown in Figure 2e,f, the cooling times for the quasi-3D NPls increase with increasing photon fluence of the pump laser, whereas they remain constant for the 2D NPls. Two processes lead to this effect, the first of which we identified as the hot photon effect and which has already been observed for bulk perovskites. ${ }^{38,39,48,49}$ In this process, the reabsorption of phonons by charge carriers, which depends strongly on the phonon density, slows down the cooling process progressively for increasing photon fluences. Second, a higher carrier density increases the dielectric permittivity of the perovskite further, leading to a stronger screening of the Fröhlich interaction and thereby a reduced cooling rate. ${ }^{19,20,31,35}$ In the $2 \mathrm{D}$ system, exhibiting a large surface-tovolume ratio, emitted optical phonons and subsequently created acoustic phonons have a much larger probability to transfer their energy into the colder medium surrounding the NPls. Thus, heat can dissipate quickly, and the probability of hot phonon reabsorption is substantially reduced. ${ }^{50,51}$ Furthermore, the increasing photoexcited carrier density can only screen the Fröhlich interaction within the thin 2D NPls, while the field lines of this interaction in the surrounding remain unaffected. Thus, the scattering rates stay consistently high even for high excitation densities.

Resonant versus Nonresonant Excitation. In the experiments presented so far, optical excitation always occurred far above the bandgap, thus creating hot charge carriers, which decay subsequently through phonon emission as depicted in Figure 4a,b. To circumvent the entire cooling process and investigate a (potential) change in the resulting dynamics, we excite the $1 \mathrm{~s}$ exciton at $E_{x}=E_{\mathrm{c}}-E_{\mathrm{b}}$ directly (Figure $4 \mathrm{~d}, \mathrm{e})$. To this end, we pumped the quasi-3D and 2D
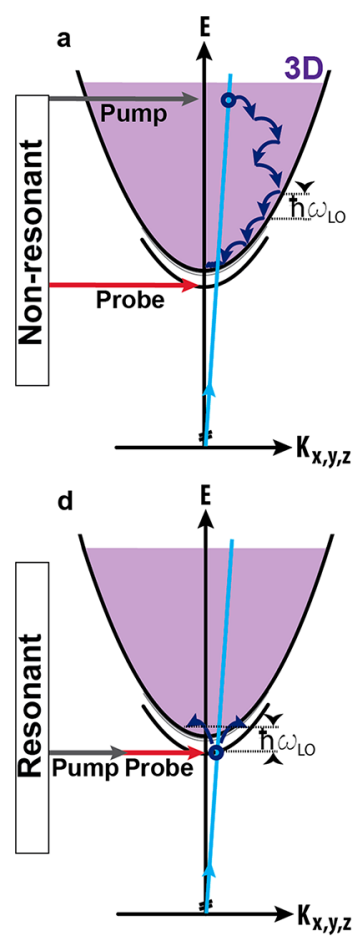

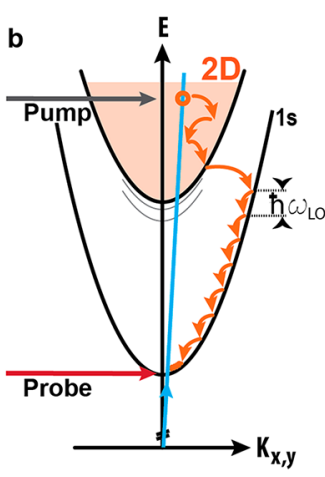

e

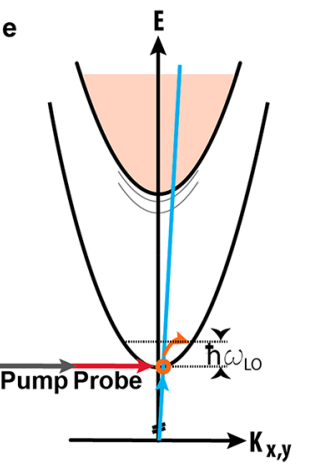

c

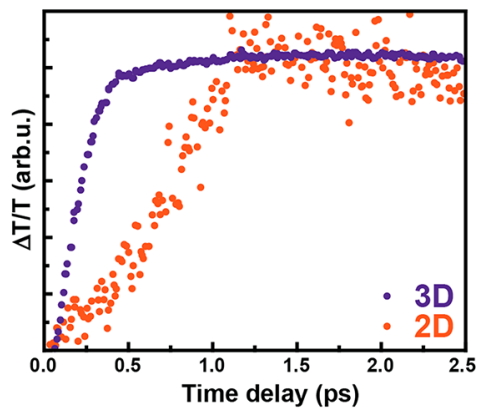

f

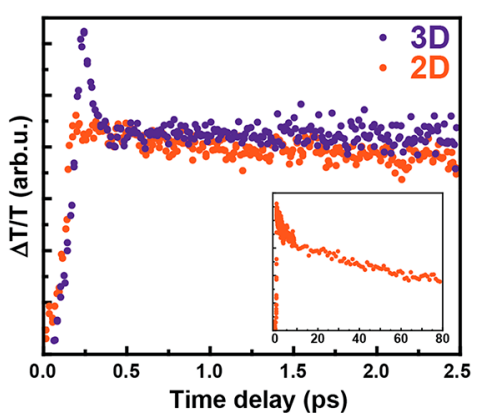

Figure 4. Resonant and nonresonant excitation of the 1s exciton transition. (a, b) Scheme of charge carrier relaxation after nonresonant (high energy) excitation of the quasi-3D (a) and 2D (b) NPls. Relaxation occurs predominantly via the emission of optical phonons. (c) Corresponding $\Delta T$ transients for the quasi-3D (purple) and 2D (orange) NPls. (d, e) Scheme of excitonic dynamics after resonant excitation of the 1s exciton transition for quasi-3D (d) and 2D (e) NPls, respectively. (f) Corresponding $\Delta T$ transients for the quasi-3D (purple) and 2D (orange) NPls. The inset in (f) shows the heating of resonantly excited "cold" 1 s excitons at longer times. 
NPl samples both resonantly with the 1 s exciton peaks at 758 and $605 \mathrm{~nm}$ and nonresonantly at 565 and $400 \mathrm{~nm}$, respectively. We then analyze the $\Delta T$ transients for the wavelengths of the probe pulse coinciding with the 1s exciton energy (Figure $4 c, f)$. For nonresonant excitation, we see that the $\Delta T$ transient of the quasi-3D sample (Figure 4c, purple data points) shows a fast initial rise followed by a slower increase to the full maximum after $1.8 \mathrm{ps}$. We ascribe these two distinct dynamical time windows to the carrier thermalization and the subsequent cooling dynamics of photoexcited carriers as discussed above. Since the 1s exciton binding energy is small for the quasi-3D NPls, carriers reaching the bottom of the continuum transitions are already within the thermal window $k T_{\mathrm{L}}$ of the $1 \mathrm{~s}$ exciton. In contrast, for nonresonant excitation of the $2 \mathrm{D} \mathrm{NPls}$, the $\Delta T$ transient detected at the $1 \mathrm{~s}$ exciton (Figure $4 c$, orange data points) shows a significantly slower increase and only one observable time regime. In order to energetically reach the optically accessible $K_{x, y}=0$ state of the $1 \mathrm{~s}$ exciton parabola, lying $230 \mathrm{meV}$ below the onset of the continuum transitions, a multitude of LO-phonons (approximately 15 LO-phonons) must be emitted. Due to the small DOS of the excitonic states as compared to the continuum states, this takes time and explains the slow rise of the $\Delta T$ transient for 2D-NPLs. For the quasi-3D NPls, the emission of only one LO-phonon is sufficient to overcome this energy difference, and only a fraction of photoexcited electron-hole pairs are expected to become converted into 1s excitons $\left(E_{3 \mathrm{D}}^{\mathrm{b}}<\right.$ $\left.k_{\mathrm{B}} T_{\mathrm{L}}\right) \cdot{ }^{41,42}$

Under resonant excitation (Figure $4 \mathrm{~d}, \mathrm{e}$ ), the $\Delta T$ transients of both samples (Figure $4 \mathrm{f}$ ) increase similarly fast with a time constant of approximately $200 \mathrm{fs}$, close to the time resolution of our experiment. For the quasi-3D NPls, the fast onset is followed by a similarly fast decay, which reduces the $\Delta T$ transient down to a value of $70 \%$ of the maximum signal in only 170 fs. As explained previously, photoexcited charge carriers initially exhibit $\delta$-function-like nonthermal distributions. As the energy difference between the $1 \mathrm{~s}$ and continuum is less than the thermal energy and comparable to that of the optical phonons in perovskite, the resonantly created "cold" excitons can be scattered by thermally available phonons into continuum states where the DOS is high. Scattered away from the light line, they no longer bleach the absorption, leading to the rapid drop in the $\Delta T / T$ signal. This dissociation of excitons has also been observed in four-wave mixing spectroscopy, which we used previously to deduce the homogeneous line width of perovskite NCs from the dephasing time $T_{2}$ of the $1 \mathrm{~s}$ exciton transition. ${ }^{24}$ The subsequent decay, which is significantly slower and constant for all observed times, is due to the recombination of excitons. In contrast, it seems as though the 2D NPls do not exhibit such a behavior. However, if we look at longer times up to 80 ps, as depicted in the inset in Figure $4 \mathrm{f}$ and in Figure S4, the decay of the $\Delta T$ transient strongly resembles that of the quasi-3D sample, albeit on a much longer time scale. In fact, the signal decays to approximately $77 \%$ of the maximum signal within 10 ps. In principle, the process is the same, as resonantly excited $K_{x, y}=0$ excitons scatter with phonons (also LO) into $K_{x, y}>0$ states on the 1s parabola as depicted in Figure 4e. However, with a binding energy larger than $200 \mathrm{meV}$, excitons cannot be scattered into the continuum, and thus thermalization can only take place with states located on the 1s exciton parabola. As the DOS here is much lower than in the continuum states, the phonon-induced heating of resonantly excited "cold" excitons occurs significantly slower in the 2D than in the quasi 3D case. Exciton recombination from the radiative window then progresses as discussed above.

\section{CONCLUSIONS}

In summary, we have compared the relaxation and recombination dynamics of photoexcited electron-hole pairs in $2 \mathrm{D}$ and $3 \mathrm{D}$ MAPI NPls by means of transient DTS. Focusing on the cooling of excited electron-hole pairs, we find striking and initially unexpected differences. For the 2D case, relaxation is significantly faster than for their $3 \mathrm{D}$ counterparts and is independent of the excitation fluence. We attribute this to a reduced screening of the Frölich interaction in the 2D system and its low dielectric surrounding resulting in enhanced and density-independent phonon emission as well as rapid dissipation of the energy from the phonon system to the surrounding medium. Additionally, we observe a rapid dissociation of "cold" excitons in the 3D system through scattering with optical phonons, an effect which is precluded in the $2 \mathrm{D}$ system due to the large exciton binding energy. These results constitute a fundamental insight into how dimensionality affects the relaxation dynamics of unbound and bound electron-hole pairs in halide perovskite NPls. Importantly, these findings will facilitate the development and choice of material parameters for optical devices relying on high speeds and cutoff frequencies, for example, photodetectors and alloptical switches. With relaxation times on the order of $0.3 \mathrm{ps}$, the $2 \mathrm{D}$ system could lead to optoelectronic devices with switching times in the $\mathrm{THz}$ regime. However, the embedding medium is highly critical to achieving these speeds.

\section{METHODS}

Synthesis of Perovskite Nanoplatelets (2D and Quasi-3D). In a typical synthesis, $10 \mathrm{~mL}$ of toluene, $0.5 \mathrm{~mL}$ of oleylamine, and 0.5 $\mathrm{mL}$ of oleic acid were added to a mixture of $0.16 \mathrm{mmol} \mathrm{MAI}$ and 0.16 mmol $\mathrm{PbI}_{2}$ precursor powders in a $20 \mathrm{~mL}$ glass bottle. The reaction mixture was subjected to tip sonication (SonoPlus HD 3100, Bandelin) at $50 \%$ of its maximum power for $30 \mathrm{~min}$. During sonication, the formation of the perovskite is evident through the change of color of the solution from yellow to black via orange and red. The as-obtained solution contains NCs of different sizes and shapes. The as-prepared colloidal solution was centrifuged at a speed of $8000 \mathrm{rpm}$ for $10 \mathrm{~min}$. Then, the supernatant which contained thin plates was separated from the $3 \mathrm{D}$ NPls present in the sediment. The sediment was washed with toluene twice and then redispersed in toluene for further studies. The colloidal solution of $3 \mathrm{ML}$ NPls was obtained by adding $200 \mu \mathrm{L}$ of the initial supernatant to $10 \mathrm{~mL}$ of toluene. The resultant colloidal solution appears orange. The morphology of the obtained NPls was characterized with a transmission electron microscope (TEM) operating at an accelerating voltage of $80-100 \mathrm{kV}$ (JEOL JEM-1011). For TEM characterization of $3 \mathrm{ML} \mathrm{NPls,} 3 \mathrm{~mL}$ of the as-obtained orange color solution was centrifuged at a speed of $14,000 \mathrm{rpm}$ for $20 \mathrm{~min}$, and then the sediment was redispersed in $0.3 \mathrm{~mL}$ of hexane.

Steady-State Optical Measurements. UV-vis absorption spectra of perovskite NPls in solution were recorded using a Varian Cary 5000 UV-vis-IR spectrometer. PL measurements were acquired with a Varian Cary Eclipse fluorescence spectrophotometer (Agilent Technologies).

Ultrafast Transient Absorption Spectroscopy. We have employed transient DTS to compare carrier relaxation and recombination dynamics in $2 \mathrm{D}$ and quasi-3D halide perovskite NPls. These NPls are dissolved as colloids in toluene and are investigated at room temperature $(300 \mathrm{~K})$. Transient differential transmission spectra were taken with a custom built transient absorption spectrometer from Newport Inc. As light source, a 1 
$\mathrm{kHz}$ femtosecond Ti:Sa amplifier system (Libra, Coherent Inc.) was used in combination with an optical parametric amplifier (Opera Solo, Coherent Inc.), providing $100 \mathrm{fs}$ laser pulses with wavelengths over a wide spectral range $(290 \mathrm{~nm}$ to $10 \mu \mathrm{m})$. The diameter of the spot size of the excitation beam was about $350 \mu \mathrm{m}$. The same laser system was used to generate a white light probe beam. The samples were measured in ambient conditions in quartz cuvettes with an optical path length of $2 \mathrm{~mm}$ and an excitation wavelength of $400 \mathrm{~nm}$ (well above the continuum absorption onset). The difference in transmission $\Delta T$ with and without the pump laser pulse present was measured as a function of the time delay $\tau$ between pump and probe pulses. For both samples and for all time delays, the differential transmission spectra shown in Figure $2 \mathrm{~b}, \mathrm{c}$ exhibit a maximum bleaching signal located spectrally at the position of the 1s exciton resonance, as determined from the linear absorption spectra (Figure 1a and Figure S3). This bleaching reaches a maximum after approximately $1 \mathrm{ps}$ and decays subsequently on a much longer time scale.

\section{ASSOCIATED CONTENT}

\section{S Supporting Information}

The Supporting Information is available free of charge on the ACS Publications website at DOI: 10.1021/acsnano.8b05029.

Detailed information on the applied Elliot model and fitting curves of the model applied to linear absorption spectra, TEM images of the perovskite NPls, laser fluences and corresponding charge carrier cooling times, differential transmission curves at maximum bleach signal and resonant excitation of the $1 \mathrm{~s}$ state (PDF)

\section{AUTHOR INFORMATION}

\section{Corresponding Authors}

*E-mail: urban@lmu.de.

*E-mail: feldmann@lmu.de.

\section{ORCID}

Lakshminarayana Polavarapu: 0000-0002-9040-5719

Alexander S. Urban: 0000-0001-6168-2509

\section{Present Address}

${ }^{\S}$ Nanospectroscopy Group, Department of Physics, LudwigMaximilians-Universität München, Amalienstr. 54, 80799 Munich, Germany

\section{Notes}

The authors declare no competing financial interest.

\section{ACKNOWLEDGMENTS}

This work was supported by the Bavarian State Ministry of Science, Research, and Arts through the grant "Solar Technologies go Hybrid" (SolTech), by the European Research Council Horizon 2020 Marie Skłodowska-Curie grant agreement COMPASS (691185) and the ERC grant agreement PINNACLE (759744), and by LMU Munich's Institutional Strategy LMUexcellent within the framework of the German Excellence Initiative. The authors would like to thank Thomas Simon for helpful discussions.

\section{REFERENCES}

(1) Stranks, S. D.; Eperon, G. E.; Grancini, G.; Menelaou, C.; Alcocer, M. J. P.; Leijtens, T.; Herz, L. M.; Petrozza, A.; Snaith, H. J. Electron-Hole Diffusion Lengths Exceeding 1 Micrometer in an Organometal Trihalide Perovskite Absorber. Science 2013, 342, 341344 .

(2) Zhou, H. P.; Chen, Q.; Li, G.; Luo, S.; Song, T. B.; Duan, H. S.; Hong, Z. R.; You, J. B.; Liu, Y. S.; Yang, Y. Interface Engineering of Highly Efficient Perovskite Solar Cells. Science 2014, 345, 542-546.
(3) De Wolf, S.; Holovsky, J.; Moon, S. J.; Loper, P.; Niesen, B.; Ledinsky, M.; Haug, F. J.; Yum, J. H.; Ballif, C. Organometallic Halide Perovskites: Sharp Optical Absorption Edge and Its Relation to Photovoltaic Performance. J. Phys. Chem. Lett. 2014, 5, 1035-1039.

(4) Saliba, M.; Matsui, T.; Seo, J. Y.; Domanski, K.; Correa-Baena, J. P.; Nazeeruddin, M. K.; Zakeeruddin, S. M.; Tress, W.; Abate, A.; Hagfeldt, A.; Gratzel, M. Cesium-Containing Triple Cation Perovskite Solar Cells: Improved Stability, Reproducibility and High Efficiency. Energy Environ. Sci. 2016, 9, 1989-1997.

(5) Akkerman, Q. A.; D’Innocenzo, V.; Accornero, S.; Scarpellini, A.; Petrozza, A.; Prato, M.; Manna, L. Tuning the Optical Properties of Cesium Lead Halide Perovskite Nanocrystals by Anion Exchange Reactions. J. Am. Chem. Soc. 2015, 137, 10276-10281.

(6) Nedelcu, G.; Protesescu, L.; Yakunin, S.; Bodnarchuk, M. I.; Grotevent, M. J.; Kovalenko, M. V. Fast Anion-Exchange in Highly Luminescent Nanocrystals of Cesium Lead Halide Perovskites ( $\left.\mathrm{CsPbX}_{3}, \mathrm{X}=\mathrm{Cl}, \mathrm{Br}, \mathrm{I}\right)$. Nano Lett. 2015, 15, 5635-5640.

(7) Xing, G. C.; Mathews, N.; Lim, S. S.; Yantara, N.; Liu, X. F.; Sabba, D.; Gratzel, M.; Mhaisalkar, S.; Sum, T. C. Low-Temperature Solution-Processed Wavelength-Tunable Perovskites for Lasing. Nat. Mater. 2014, 13, 476-480.

(8) Saparov, B.; Mitzi, D. B. Organic-Inorganic Perovskites: Structural Versatility for Functional Materials Design. Chem. Rev. 2016, 116, 4558-4596.

(9) Kovalenko, M. V.; Protesescu, L.; Bodnarchuk, M. I. Properties and Potential Optoelectronic Applications of Lead Halide Perovskite Nanocrystals. Science 2017, 358, 745-750.

(10) Koscher, B. A.; Swabeck, J. K.; Bronstein, N. D.; Alivisatos, A. P. Essentially Trap-Free $\mathrm{CsPbBr}_{3}$ Colloidal Nanocrystals by Postsynthetic Thiocyanate Surface Treatment. J. Am. Chem. Soc. 2017, 139, 6566-6569.

(11) Sichert, J. A.; Tong, Y.; Mutz, N.; Vollmer, M.; Fischer, S.; Milowska, K. Z.; Cortadella, R. G.; Nickel, B.; Cardenas-Daw, C.; Stolarczyk, J. K.; Urban, A. S.; Feldmann, J. Quantum Size Effect in Organometal Halide Perovskite Nanoplatelets. Nano Lett. 2015, 15, 6521-6527.

(12) Hintermayr, V. A.; Richter, A. F.; Ehrat, F.; Döblinger, M.; Vanderlinden, W.; Sichert, J. A.; Tong, Y.; Polavarapu, L.; Feldmann, J.; Urban, A. S. Tuning the Optical Properties of Perovskite Nanoplatelets through Composition and Thickness by LigandAssisted Exfoliation. Adv. Mater. 2016, 28, 9478-9485.

(13) Akkerman, Q. A.; Motti, S. G.; Kandada, A. R. S.; Mosconi, E.; D'Innocenzo, V.; Bertoni, G.; Marras, S.; Kamino, B. A.; Miranda, L.; De Angelis, F.; Petrozza, A.; Prato, M.; Manna, L. Solution Synthesis Approach to Colloidal Cesium Lead Halide Perovskite Nanoplatelets with Monolayer-Level Thickness Control. J. Am. Chem. Soc. 2016, $138,1010-1016$

(14) Liu, J.; Xue, Y.; Wang, Z.; Xu, Z.-Q.; Zheng, C.; Weber, B.; Song, J.; Wang, Y.; Lu, Y.; Zhang, Y.; Bao, Q. Two-Dimensional $\mathrm{CH}_{3} \mathrm{NH}_{3} \mathrm{PbI}_{3}$ Perovskite: Synthesis and Optoelectronic Application. ACS Nano 2016, 10, 3536-3542.

(15) Weidman, M. C.; Goodman, A. J.; Tisdale, W. A. Colloidal Halide Perovskite Nanoplatelets: An Exciting New Class of Semiconductor Nanomaterials. Chem. Mater. 2017, 29, 5019-5030.

(16) Polavarapu, L.; Nickel, B.; Feldmann, J.; Urban, A. S. Advances in Quantum-Confined Perovskite Nanocrystals for Optoelectronics. Adv. Energy Mater. 2017, 7, 1700267.

(17) Wang, Q.; Liu, X.-D.; Qiu, Y.-H.; Chen, K.; Zhou, L.; Wang, Q.-Q. Quantum Confinement Effect and Exciton Binding Energy of Layered Perovskite Nanoplatelets. AIP Adv. 2018, 8, 025108.

(18) Yang, D.; Zou, Y.; Li, P.; Liu, Q.; Wu, L.; Hu, H.; Xu, Y.; Sun, B.; Zhang, Q.; Lee, S.-T. Large-Scale Synthesis of Ultrathin Cesium Lead Bromide Perovskite Nanoplates with Precisely Tunable Dimensions and Their Application in Blue Light-Emitting Diodes. Nano Energy 2018, 47, 235-242.

(19) Sohier, T.; Calandra, M.; Mauri, F. Two-Dimensional Frohlich Interaction in Transition-Metal Dichalcogenide Monolayers: Theoretical Modeling and First-Principles Calculations. Phys. Rev. B: Condens. Matter Mater. Phys. 2017, 96, 085415. 
(20) Shah, J. Hot Carriers in Semiconductor Nanostructures: Physics and Applications; Academic Press, Inc.: San Diego, CA, 1992.

(21) Ma, J.; Wang, L. W. Nanoscale Charge Localization Induced by Random Orientations of Organic Molecules in Hybrid Perovskite $\mathrm{CH}_{3} \mathrm{NH}_{3} \mathrm{PbI}_{3}$. Nano Lett. 2015, 15, 248-253.

(22) Bohn, B. J.; Tong, Y.; Gramlich, M.; Lai, M. L.; Döblinger, M.; Wang, K.; Hoye, R. L. Z.; Müller-Buschbaum, P.; Stranks, S. D.; Urban, A. S.; Polavarapu, L.; Feldmann, J. Boosting Tunable Blue Luminescence of Halide Perovskite Nanoplatelets through Postsynthetic Surface Trap Repair. Nano Lett. 2018, 18, 5231-5238.

(23) Herz, L. M. Charge-Carrier Dynamics in Organic-Inorganic Metal Halide Perovskites. Annu. Rev. Phys. Chem. 2016, 67, 65-89.

(24) Bohn, B. J.; Simon, T.; Gramlich, M.; Richter, A. F.; Polavarapu, L.; Urban, A. S.; Feldmann, J. Dephasing and Quantum Beating of Excitons in Methylammonium Lead Iodide Perovskite Nanoplatelets. ACS Photonics 2018, 5, 648-654.

(25) Miyata, A.; Mitioglu, A.; Plochocka, P.; Portugall, O.; Wang, J. T.-W.; Stranks, S. D.; Snaith, H. J.; Nicholas, R. J. Direct Measurement of the Exciton Binding Energy and Effective Masses for Charge Carriers in Organic-Inorganic Tri-Halide Perovskites. Nat. Phys. 2015, 11, 582-587.

(26) Ishihara, T.; Takahashi, J.; Goto, T. Optical Properties Due to Electronic Transitions in Two-Dimensional Semiconductors $\left(\mathrm{C}_{\mathrm{n}} \mathrm{h}_{2 \mathrm{n}+1} \mathrm{nh}_{3}\right)_{2} \mathrm{pbi}_{4}$. Phys. Rev. B: Condens. Matter Mater. Phys. 1990, 42, 11099-11107.

(27) Hong, X.; Ishihara, T.; Nurmikko, A. V. Dielectric Confinement Effect on Excitons in $\mathrm{PbI}_{4}$-Based Layered Semiconductors. Phys. Rev. B: Condens. Matter Mater. Phys. 1992, 45, 6961-6964.

(28) Hirasawa, M.; Ishihara, T.; Goto, T.; Sasaki, S.; Uchida, K.; Miura, N. Magnetoreflection of the Lowest Exciton in a Layered Perovskite-Type Compound $\left(\mathrm{C}_{10} \mathrm{H}_{21} \mathrm{NH}_{3}\right)_{2} \mathrm{PbI}_{4}$. Solid State Commun. 1993, 86, 479-483.

(29) Feldmann, J.; Peter, G.; Gobel, E. O.; Dawson, P.; Moore, K.; Foxon, C.; Elliott, R. J. Linewidth Dependence of Radiative Exciton Lifetimes in Quantum-Wells. Phys. Rev. Lett. 1987, 59, 2337-2340.

(30) Tessier, M. D.; Javaux, C.; Maksimovic, I.; Loriette, V.; Dubertret, B. Spectroscopy of Single CdSe Nanoplatelets. ACS Nano 2012, 6, 6751-6758.

(31) Shah, J. Ultrafast Spectroscopy of Semiconductors and Semiconductor Nanostructures; Springer: Berlin, 1996; p xv.

(32) Richter, J. M.; Branchi, F.; Camargo, F. V. D.; Zhao, B. D.; Friend, R. H.; Cerullo, G.; Deschler, F. Ultrafast Carrier Thermalization in Lead Iodide Perovskite Probed with Two-Dimensional Electronic Spectroscopy. Nat. Commun. 2017, 8, 376.

(33) Potz, W. Hot-Phonon Effects in Bulk GaAs. Phys. Rev. B: Condens. Matter Mater. Phys. 1987, 36, 5016-5019.

(34) Brinkman, W. F.; Rice, T. M. Electron-Hole Liquids in Semiconductors. Phys. Rev. B 1973, 7, 1508-1523.

(35) Skolnick, M. S.; Nash, K. J.; Tapster, P. R.; Mowbray, D. J.; Bass, S. J.; Pitt, A. D. Free-Carrier Screening of the Interaction between Excitons and Longitudinal-Optical Phonons in $\operatorname{In}_{\mathrm{x}} \mathrm{Ga}_{1-\mathrm{X}} \mathrm{As}-$ InP Quantum-Wells. Phys. Rev. B: Condens. Matter Mater. Phys. 1987, $35,5925-5928$

(36) Kaasbjerg, K.; Bhargavi, K. S.; Kubakaddi, S. S. Hot-Electron Cooling by Acoustic and Optical Phonons in Monolayers of $\mathrm{MoS}_{2}$ and Other Transition-Metal Dichalcogenides. Phys. Rev. B: Condens. Matter Mater. Phys. 2014, 90, 165436.

(37) Manser, J. S.; Kamat, P. V. Band Filling with Free Charge Carriers in Organonietal Halide Perovskites. Nat. Photonics 2014, 8, 737-743.

(38) Price, M. B.; Butkus, J.; Jellicoe, T. C.; Sadhanala, A.; Briane, A.; Halpert, J. E.; Broch, K.; Hodgkiss, J. M.; Friend, R. H.; Deschler, F. Hot-Carrier Cooling and Photoinduced Refractive Index Changes in Organic-Inorganic Lead Halide Perovskites. Nat. Commun. 2015, 6, 8420.

(39) Yang, Y.; Ostrowski, D. P.; France, R. M.; Zhu, K.; van de Lagemaat, J.; Luther, J. M.; Beard, M. C. Observation of a HotPhonon Bottleneck in Lead-Iodide Perovskites. Nat. Photonics 2016, $10,53-59$.
(40) Eperon, G. E.; Jedlicka, E.; Ginger, D. S. Biexciton Auger Recombination Differs in Hybrid and Inorganic Halide Perovskite Quantum Dots. J. Phys. Chem. Lett. 2018, 9, 104-109.

(41) Wright, A. D.; Verdi, C.; Milot, R. L.; Eperon, G. E.; PerezOsorio, M. A.; Snaith, H. J.; Giustino, F.; Johnston, M. B.; Herz, L. M. Electron-Phonon Coupling in Hybrid Lead Halide Perovskites. Nat. Commun. 2016, 7, 11755.

(42) Sendner, M.; Nayak, P. K.; Egger, D. A.; Beck, S.; Müller, C.; Epding, B.; Kowalsky, W.; Kronik, L.; Snaith, H. J.; Pucci, A.; Lovrinčić, R. Optical Phonons in Methylammonium Lead Halide Perovskites and Implications for Charge Transport. Mater. Horiz. 2016, 3, 613-620.

(43) Heitz, R.; Kalburge, A.; Xie, Q.; Grundmann, M.; Chen, P.; Hoffmann, A.; Madhukar, A.; Bimberg, D. Excited States and Energy Relaxation in Stacked InAs/GaAs Quantum Dots. Phys. Rev. B: Condens. Matter Mater. Phys. 1998, 57, 9050-9060.

(44) Guyot-Sionnest, P.; Shim, M.; Matranga, C.; Hines, M. Intraband Relaxation in CdSe Quantum Dots. Phys. Rev. B: Condens. Matter Mater. Phys. 1999, 60, R2181-R2184.

(45) Pandey, A.; Guyot-Sionnest, P. Slow Electron Cooling in Colloidal Quantum Dots. Science 2008, 322, 929-932.

(46) Feldmann, J.; Cundiff, S. T.; Arzberger, M.; Bohm, G.; Abstreiter, G. Carrier Capture into InAs/GaAs Quantum Dots via Multiple Optical Phonon Emission. J. Appl. Phys. 2001, 89, 11801183.

(47) Li, M. J.; Bhaumik, S.; Goh, T. W.; Kumar, M. S.; Yantara, N.; Gratzel, M.; Mhaisalkar, S.; Mathews, N.; Sum, T. C. Slow Cooling and Highly Efficient Extraction of Hot Carriers in Colloidal Perovskite Nanocrystals. Nat. Commun. 2017, 8, 14350.

(48) Deschler, F.; Price, M.; Pathak, S.; Klintberg, L. E.; Jarausch, D. D.; Higler, R.; Huttner, S.; Leijtens, T.; Stranks, S. D.; Snaith, H. J.; Atature, M.; Phillips, R. T.; Friend, R. H. High Photoluminescence Efficiency and Optically Pumped Lasing in Solution-Processed Mixed Halide Perovskite Semiconductors. J. Phys. Chem. Lett. 2014, 5, $1421-1426$

(49) Fu, J. H.; Xu, Q.; Han, G. F.; Wu, B.; Huan, C. H. A.; Leek, M. L.; Sum, T. C. Hot Carrier Cooling Mechanisms in Halide Perovskites. Nat. Commun. 2017, 8, 1300.

(50) Bao, H.; Habenicht, B. F.; Prezhdo, O. V.; Ruan, X. L. Temperature Dependence of Hot-Carrier Relaxation in Pbse Nanocrystals: An $A b$ Initio Study. Phys. Rev. B: Condens. Matter Mater. Phys. 2009, 79, 235306.

(51) Cooney, R. R.; Sewall, S. L.; Dias, E. A.; Sagar, D. M.; Anderson, K. E. H.; Kambhampati, P. Unified Picture of Electron and Hole Relaxation Pathways in Semiconductor Quantum Dots. Phys. Rev. B: Condens. Matter Mater. Phys. 2007, 75, 245311. 